\title{
Ultraschallkurse seit dem 01.10.21 gemäß Protokollnotiz 4 der Ultraschallvereinbarung
}

Eine Fortführung der Pandemieregelung für Ultraschallkurse gemäß Protokollnotiz 3 der Ultraschallvereinbarung über den 30. September 2021 hinaus wurde kürzlich von den Gesetzlichen Krankenkassen abgelehnt. Seit dem 1. Oktober 2021 gelten für die Durchführung KV-konformer Ultraschallkurse wieder die Vorgaben, die gemäß der Ultraschallvereinbarung (USV) in der Zeit vor der Corona-Pandemie gegolten haben. Das betrifft auch die nun wieder in Präsenz durchzuführenden praktischen Übungen.

Die seit dem 1. Oktober 2021 gültige Protokollnotiz 4 der USV enthält jedoch einige Neuerungen, die im Folgenden dargestellt werden:

Die Kurstage und Module müssen nicht unmittelbar aufeinander folgen. Die Zeit zwischen dem ersten und letzten Kurstag soll 4 Wochen - gegebenenfalls einschließlich der angrenzenden Wochenenden nicht überschreiten.

Der theoretische Teil der Ultraschallkurse nach §6 kann zunächst für die Dauer von 3 Jahren in Teilen oder insgesamt unter folgenden Voraussetzungen als Onlinekurs abgehalten werden: a) Die Möglichkeit einer Interaktion zwischen Kursleiter*innen und Kursteilnehmenden ist durch eine geeignete Plattform und darüber angebotene Anwendungen (z. B. Fragefunktion für alle Teilnehmer) zu gewährleisten.

b) Die Teilnahme an allen Kursbestandteilen muss vom Kursleiter oder der Kursleiterin überprüfbar sein.

c) Die Anzahl der Teilnehmenden ist auf 30 je Kursleiter*in beschränkt.

d) Der Onlinekurs erfolgt in sinnvoll in sich abgeschlossenen, themenbezogenen Blöcken.

Die Kassenärztliche Vereinigung dokumentiert die Art des besuchten theoretischen Teils der Ultraschallkurse (Präsenzkurs, Onlinekurs oder Mischform) und das Ergebnis des KV-Kolloquiums gemäß §6 Abs. 1 Buchstabe c der USV (bestanden, nicht bestanden). Die Kassenärztlichen Vereinigungen informieren die Kassenärztliche Bundesvereinigung jeweils zum 30.04. des Folgejahres über den Nachweis der fachlichen Befähigungen nach §6 im Rahmen der erteilten Genehmigungen wie folgt:

- Anzahl der bestandenen und der nicht bestandenen Kolloquien, bei denen der Nachweis der fachlichen Befähigung bezüglich des theoretischen Kursteils aus Präsenzkursen stammt,
- Anzahl der bestandenen und der nicht bestandenen Kolloquien, bei denen der Nachweis der fachlichen Befähigung bezüglich des theoretischen Kursteils aus Präsenz- und Onlinekursen stammt,

- Anzahl der bestandenen und der nicht bestandenen Kolloquien, bei denen der Nachweis der fachlichen Befähigung bezüglich des theoretischen Kursteils aus Onlinekursen stammt.

Nach 3 Jahren entscheiden die Vertragspartner KBV und Spitzenverband Bund der Krankenkassen anhand der übermittelten Daten, ob, bzw. in welcher Form der theoretische Kursteil online fortgeführt werden soll.

Bei diesen Neuerungen handelt es sich um eine Erweiterung der Möglichkeiten künftige Ultraschallkurse zu gestalten. Damit ist keine Einschränkung der bisherigen Kurskonzepte verbunden. Alle Präsenzkurse, die vor Corona KV-konform durchgeführt wurden, können selbstverständlich auch weiterhin genauso veranstaltet werden.

Dr. Hans Worlicek, Leiter der Kommission Ultraschall in der Praxis

Prof Dr. Josef Menzel, Präsident der DEGUM 